\section{$\checkmark$ Research Square}

Preprints are preliminary reports that have not undergone peer review.

They should not be considered conclusive, used to inform clinical practice, or referenced by the media as validated information.

\title{
Ultrasound-Guided Microwave Ablation for Benign Thyroid Nodules: A Retrospective Analysis of Safety and Efficacy Factors
}

\section{Baoying Xia}

West China Hospital of Sichuan University

Boyang Yu

West China Hospital of Sichuan University

\section{Xiaofei Wang}

West China Hospital of Sichuan University

Yu Ma

West China Hospital of Sichuan University

\section{Feng Liu}

West China Hospital of Sichuan University

Yanping Gong

West China Hospital of Sichuan University

Xiuhe Zou

West China Hospital of Sichuan University

Jianyong Lei

West China Hospital of Sichuan University

\section{Anping Su}

West China Hospital of Sichuan University

Tao Wei

West China Hospital of Sichuan University

Jingqiang Zhu

West China Hospital of Sichuan University

Qiang Lu

West China Hospital of Sichuan University

Zhihui Li ( $D$ 452863580@qq.com )

West China Hospital of Sichuan University

\section{Research Article}

Keywords: Thyroid nodule, Microwave ablation, Volume reduction ratio, Initial volume 
Posted Date: January 7th, 2021

DOl: https://doi.org/10.21203/rs.3.rs-137725/v1

License: (9) This work is licensed under a Creative Commons Attribution 4.0 International License. Read Full License

Version of Record: A version of this preprint was published at BMC Surgery on August 3rd, 2021. See the published version at https://doi.org/10.1186/s12893-021-01312-1. 


\section{Abstract}

Objective

Microwave ablation (MWA) is a minimally invasive technique for the treatment of benign thyroid nodules. The purpose of this study was to evaluate the efficacy and safety of ultrasound-guided microwave ablation in the treatment of benign thyroid nodules, and to provide reference for future clinical work.

Methods

This study retrospectively analyzed the patients who received ultrasound-guided microwave ablation for benign thyroid nodules in our hospital from October 2018 to March 2020. A total of 214 patients were included in the study. We assessed thyroid volume changes (represented by volume reduction ratio VRR), the serum levels of triiodide thyroid hormone (T3), thyroxine (T4) and thyrotropin (TSH) and complications after MWA treatment.

Results

There were a total of 306 nodules in 214 patients, including 183 (85.51\%) females and $31(14.49 \%)$ males. The median diameter and volume of the nodule were $33 \mathrm{~mm}$ and $8.01 \mathrm{ml}$. The volume reduction ratioهVRR囚at 1 month, 3 months, 6 months and 12 months were $40.79 \%, 60.37 \%, 74.59 \%$ and $85.60 \%$, respectively. $\otimes \mathrm{P}<0.05)$ In addition, initial nodular volume were the influencing factors of volume reduction ratio.

\section{Conclusions}

Ultrasound-guided MWA is effective and safe in the treatment of benign thyroid nodules. In addition, it has little damage to surrounding tissues and no effect on thyroid function. Especially for the initial small volume of nodules, treatment is better. Therefore, ultrasound-guided thyroid MWA can be used as one of the main clinical treatment methods for benign thyroid nodules.

\section{Introduction}

Thyroid nodules are very common in the population and account for $65 \%-95 \%$ of all thyroid diseases. ${ }^{[1-}$ ${ }^{3]}$ Thyroid nodules are often detected by color Doppler ultrasound. More than $90 \%$ of thyroid nodules are benign. ${ }^{[4]}$ Current guidelines indicate that some asymptomatic thyroid nodules may be continued to be observed, and that further treatment is often required when symptomatic compression or cosmetic effects occur. Previously, the treatment of benign thyroid nodules mainly through surgery and levothyroxine therapy. ${ }^{[5]}$ On the one hand, the efficacy of levothyroxine is not significant; on the other hand, the surgical treatment has many disadvantages such as large trauma and permanent surgical scar. Therefore, benign thyroid nodule minimally invasive surgery such as thermal ablation came into being. 
Thermal ablation includes laser ablation, radiofrequency ablation and microwave ablation, among which radiofrequency ablation is the most common. ${ }^{[6]}$ Radiofrequency ablation has been routinely used for the treatment of liver, vascular and other diseases. ${ }^{[7]}$ However, although good therapeutic effects have been achieved for cystic thyroid nodules, the therapeutic effects for solid nodules are not very significant. ${ }^{[8]}$ As a minimally invasive technique, microwave ablation has been gradually developed in clinic due to its good effect in the treatment of benign thyroid nodules. Therefore, this study retrospectively analyzed the volume reduction ratio(VRR) of thyroid nodules after ultrasound-guided microwave ablation to explore the therapeutic effect and safety of microwave ablation on benign thyroid nodules.

\section{Materials And Methods}

\section{Clinical data}

This retrospective study selected patients who underwent ultrasound guided microwave ablation of benign thyroid nodules at West China Hospital of Sichuan University from October 2018 to March 2020. The inclusion criteria were: (1)Consistent with the diagnosis of benign thyroid nodules (ultrasound, CT or fine needle aspiration); $\mathbb{2} 2 \triangle T$ These patients are in line with the indications of ultrasunion-guided microwave ablation for the treatment of benign thyroid nodules $\mathbb{Q} \otimes \mathbf{Z}$ Local residents or long-term residents of the city and have good compliance. The exclusion criteria were: $₫ 1 \otimes P a t i e n t s$ with confirmed thyroid cancer; $₫ 2$ $\square$ Patients with benign thyroid nodules underwent surgical resection $\varangle 3 \square$ Associated with severe disease

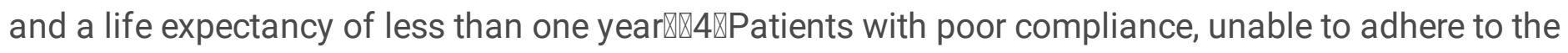
review.

\section{Data collection}

Collect basic information of patients, including name, admission number, gender, age, etc. Preoperative benign thyroid nodules, size, nature, duration of surgery, ablation power, complications, average length of stay. The size of benign thyroid nodules and the levels of TSH, FT3 and FT4 in plasma at 1, 3, 6 and 12 months after the operation.

Evaluation and follow-up

The nodules were divided into three types: cystic nodules (liquid composition $>90 \%$ ); Solid nodules (liquid composition $\leq 10 \%)$; the rest were cystic and solid mixture nodules. Small nodules were defined as initial volume $\leq 10 \mathrm{~mL}$. ${ }^{[9]}$

The therapeutic effect of ultrasound follow-up at 1, 3, 6 and 12 months after treatment was calculated, and the nodule volume $(\mathrm{V})$ and lesion volume reduction ratio $(\mathrm{VRR})$ were calculated: $\mathrm{V}=$ length $(\mathrm{cm})$ * width $(\mathrm{cm}) *$ height $(\mathrm{cm}) \star 0.524(\mathrm{ml})$. Volume reduction ratio $(V R R)$, that is the percentage of the reduction in the nodule's volume during following up compared to the initial volume: VRR $=(V O-V x) / V O * 100 \%$. The volume reduction ratio (VRR) of benign thyroid nodules $>50 \%$ in half a year was considered effective in microwave ablation treatment. ${ }^{[10]}$ 


\section{Statistical analysis}

We used statistical software SPSS 23.0 to analyze the data. The Kruskal-Wallis rank sum test was used to analyze the significance difference of skewed distribution data. Chi-square test was used to compare categorical variables. Multivariate analysis of microwave ablation effect was performed using binary Logistic regression model. The results are presented as odds ratios (ORs) with a $95 \%$ confidence interval $(\mathrm{Cl})$. The difference is statistically significant when $P$ value is less than 0.05 . Furthermore, we use Origin 9.0 to draw the statistical graph.

All the above methods are in accordance with the relevant guidelines and regulations.

\section{Results}

General information

A total of 214 patients with benign thyroid nodule treated by microwave ablation were included in this study, including 31 males and 183 females. The median age was $45.84 \pm 14.32(11-91)$ years. The duration of surgery for patients undergoing microwave ablation is approximately 7 minutes and 25 seconds and the average ablation power is $30.52(30-40) \mathrm{w}$, with an average length of stay of 2(1-7) days. The median follow-up time in this study was $10.12 \pm 2.78$ months. There were 148 cases of single nodules and 66 cases of multiple nodules.

Benign thyroid nodule characteristics

In this study, 306 thyroid nodules were ablated by microwave in 214 patients. The nodule had a median maximum diameter of $33 \mathrm{~mm}$ and a mean volume of $8.01 \mathrm{ml}$. The number of nodules in the lower thyroid lobe was the largest, accounting for $37.58 \%(115 / 306)$. The number of cystic nodules, solid nodules and mixed nodules was $26(8.50 \%), 78(25.49 \%)$ and $202(66.01 \%)$, respectively. The volume of nodules (less than $10 \mathrm{~mL}$ ) before microwave ablation was dominated by small nodules, accounting for $58.17 \%$ (178/306). ( Table 10 
Table 1

Profiles of thyroid nodules treated with MWA

\begin{tabular}{|llll|}
\hline Characteristic & & All nodules $(\mathrm{N}=306)$ & Percentage(\%) \\
\hline Largest diameter $(\mathrm{mm})$ & & $33(4-70)$ & \\
\hline Volume $(\mathrm{ml})$ & & $8.01(2.25-16.98)$ & \\
\hline Location & upper lobe & 94 & 30.72 \\
\hline & middle lobe & 97 & 31.70 \\
\hline Solidity & lower lobe & 115 & 37.58 \\
\hline & cystic & 26 & 8.50 \\
\hline & Solid & 78 & 25.49 \\
\hline Volume $(\mathrm{ml})$ & Mixed & 202 & 66.01 \\
& $\mathrm{v} \leq 10$ & 178 & 58.17 \\
\hline & $\mathrm{v}>10$ & 128 & 41.83 \\
\hline
\end{tabular}

Efficacy evaluation

After microwave ablation(MWA), the volume reduction ratio(VRR) of benign thyroid nodules increased over time. (Fig. 1) The annual overall average VRR reached $85.60 \%$. In our study, we found that ultrasound-guided microwave ablation has a better therapeutic effect on cystic nodules. However, there was no significant difference in volume reduction ratio between different thyroid nodule types (cystic, solid, mixed) 6 months after microwave ablation $(P>0.05)$. ( Table 2,Fig. 2) In addition, we found that the mean hormone levels of TSH, FT3 and FT4 in postoperative thyroid function indexes were all within the normal range. Moreover, the levels of triiodide thyroid hormone (T3) and thyroxine (T4) were not significantly different before and after surgery, while the levels of thyrotropin (TSH) were statistically different. (Table 3, Fig. 3) In univariate analysis, nodule initial volume was found to be statistically significant with VRR. (Table 4) In binary Logistic regression analysis, we found that the initial volume of thyroid nodules was an independent risk factor for the treatment of benign thyroid nodules by microwave ablation. $(P<0.05)$. (Table 5) 
Table 2

VRR of different nodule types

\begin{tabular}{|lllll|}
\hline & 1 month & 3months & 6months & 12months \\
\hline Total & 40.79 & 60.37 & 74.59 & 85.60 \\
\hline Solidity & & & & \\
\hline Cystic & 46.64 & 68.72 & 81.21 & 88.12 \\
\hline Solid & 35.59 & 58.19 & 70.46 & 84.08 \\
\hline Mixed & 43.50 & 62.28 & 75.11 & 86.24 \\
\hline$P$ value & 0.001 & 0.008 & 0.140 & 0.389 \\
\hline
\end{tabular}

Table 3

Thyroid hormone changes during follow-up period

\begin{tabular}{|llccccc|}
\hline & Before & 1 month & 3 months & 6 months & 12 months & P value \\
\hline TSH & $2.24 \pm 2.34$ & $2.43 \pm 1.94$ & $2.65 \pm 1.96$ & $2.72 \pm 1.54$ & $2.78 \pm 1.31$ & 0.026 \\
$(\mathrm{Mu} / \mathrm{L})$ & & & & & & \\
\hline $\begin{array}{l}\text { FT3 } \\
\text { (pmol/L) }\end{array}$ & $5.07 \pm 1.40$ & $4.77 \pm 1.07$ & $4.51 \pm 1.62$ & $4.32 \pm 1.32$ & $4.13 \pm 0.73$ & 0.445 \\
\hline $\begin{array}{l}\text { FT4 } \\
\text { (pmol/L) }\end{array}$ & $17.09 \pm 5.52$ & $16.09 \pm 2.71$ & $15.64 \pm 2.91$ & $15.08 \pm 2.44$ & $14.83 \pm 1.65$ & 0.447 \\
\hline
\end{tabular}


Table 4

Single factor analysis of MWA ablation effect

\begin{tabular}{|c|c|c|c|c|}
\hline Characteristics & VRR】50\% & VRR $\leq 50 \%$ & $x^{2}$ & $P$ \\
\hline Gender & & & 1.668 & 0.197 \\
\hline Male & 40 & 4 & & \\
\hline Female & 214 & 48 & & \\
\hline Age & & & 0.115 & 0.735 \\
\hline$\leq 60$ & 210 & 44 & & \\
\hline$\nabla 60$ & 44 & 8 & & \\
\hline Location & & & 1.421 & 0.491 \\
\hline upper & 81 & 13 & & \\
\hline middle & 81 & 16 & & \\
\hline lower & 92 & 23 & & \\
\hline Solidity & & & 0.944 & 0.624 \\
\hline Cyst & 20 & 6 & & \\
\hline Solid & 62 & 16 & & \\
\hline Mixed & 168 & 34 & & \\
\hline Initial volume & & & 4.340 & 0.037 \\
\hline$\leq 10 \mathrm{~mL}$ & 141 & 37 & & \\
\hline$\nabla 10 \mathrm{~mL}$ & 113 & 15 & & \\
\hline
\end{tabular}

Table 5

Logistic regression analysis of MWA ablation effect

\begin{tabular}{|lllllll|}
\hline Variable & B & $\boldsymbol{\beta}$ & Wald & $\mathbf{P}$ & OR & 95\%Cl \\
\hline Initial volume & -0.681 & 0.331 & 4.236 & 0.040 & 0.506 & $(0.264,0.968)$ \\
\hline$(\leq 10 \mathrm{~mL},>10 \mathrm{ml})$ & & & & & \\
\hline constant & 2.019 & 0.275 & 53.998 & 0.000 & 7.533 & \\
\hline
\end{tabular}

Safety assessment

Among 214 patients treated with MWA,none had serious complications. However, one patient developed intracapsular hemorrhage and recovered within a month. Another case developed recurrent laryngeal nerve injury but recovered within two months. 


\section{Discussion}

In this study, we retrospectively analyzed 214 patients who underwent ultrasound - guided microwave ablation. After $10.12 \pm 2.78$ months of follow-up, the volume reduction ratio(VRR) of benign thyroid nodules was up to $85.60 \%$. Univariate analysis showed that there was a statistical difference between the initial volume of nodules and the effect of microwave ablation. Logistic regression analysis showed that microwave ablation had a good effect on the initial volume of nodules less than or equal to $10 \mathrm{ml}$. Furthermore, the serum levels of triiodide thyroid hormone (T3), thyroxine (T4) and thyrotropin (TSH) were all within the normal range, indicating that microwave ablation would not impair thyroid function.

The therapeutic effect of microwave ablation has been proved clinically at home and abroad. It has a good therapeutic effect on benign thyroid nodules. Numerous studies have shown that the volume reduction ratio after microwave ablation can reach $24.0-51 \%, 54.8-75.1 \%, 68.7-85.2 \%, 75.8-96.4 \%$ respectively after $1,3,6$ and 12 months. ${ }^{[1,5,11-14]}$ Our study showed similar outcomes.

Our results are consistent with those reported by Liu YJ, Yue W, Wu W et al. ${ }^{[13,15,16]}$, none of the patients treated with microwave ablation had serious complications. Only one case of intrathyroid hemorrhage and postoperative hoarseness occurred in our study, but all recovered 2 months after the MWA. Hu et al. ${ }^{[12]}$ found that $2.3 \%$ of the sound changes in the study followed microwave ablation, but all recovered within two weeks. Therefore, it can be seen that microwave ablation(MWA)for benign thyroid class has the characteristics of fewer complications and easier recovery.

In our study, Logistic regression analysis showed that the initial volume of nodules, especially small nodules (volume $<10 \mathrm{ml}$ ), was a risk factor for the effect of microwave ablation. Cesareo $\mathrm{R}$ et al. ${ }^{[17]}$ obtained similar results in their study, suggesting that ablation can effectively reduce benign thyroid nodules, especially small ones. Lee GM et al. ${ }^{[10]}$ found that ablation was more effective in the treatment of small nodules with a volume less than $4 \mathrm{ml} .(P=0.030)$

Heck K et al. ${ }^{[18]}$ proposed that the serum levels of triiodothyroid hormone (T3), thyroxine (T4), thyrotrophin (TSH), thyroglobulin (Tg), anti-TG, thyrotrophin receptor (TRAb), and thyroid peroxidase (antiTPO) showed no significant changes after the microwave ablation and the follow-up of half a year. Erturk MS et al. ${ }^{[19]}$ pointed out that the effect of MWA on thyroid function had no significant difference at 6 months, but the effect was significant at 24 hours. In our study, we reached the same conclusion that the serum levels of triiodide thyroid hormone (T3), thyroxine (T4) and thyrotropin (TSH) after microwave ablation were all within the normal level and decreased compared with that before surgery. Therefore, we have reason to believe that microwave ablation has a good effect and has no effect on thyroid function.

This study still has some limitations. First, this was a retrospective study, with no control group. Second, the follow-up time in this study was not long enough so that the volume reduction ratio reached a plateau value. Third, symptom scores and cosmetic scores were not used for quantitative evaluation. Therefore, long-term, quantitative and prospective studies are needed to further verify the conclusions in the future. 


\section{Conclusion}

In summary, our study shows that ultrasound-guided microwave ablation is effective and safe in the treatment of benign thyroid nodules. In addition, microwave ablation has a good effect on different types of thyroid nodules (cystic, solid and mixed). Especially for the initial small volume of nodules, treatment is better. Microwave ablation(MWA) is characterized by better protection of thyroid function, fewer complications and superior esthetic results. In clinical practice, we suggest that ultrasound-guided microwave ablation should be the first choice for the treatment of benign thyroid nodules.

\section{Declarations}

Ethics approval and consent to participate

This research is based on the Declaration of Helsinki, and the study was approved by Ethics Committee on Biomedical Research, West China Hospital of Sichuan University (approval number: 2020599).

Consent to publish

Written informed consent for publication of their clinical details was obtained from all patients.

Availability of data and materials

The datasets used and analyzed during the current study are available from the corresponding author on reasonable request.

Competing interests

The authors have no competing interest to declare.

Funding

Not applicable.

Acknowledgement

Not applicable.

Authors' information

Baoying Xia and Boyang Yu contribute equally to this paper.

Affiliations

1. Surgical Treatment center of Thyroid/Parathyroid Diseases, West China Hospital, Sichuan University(Chengdu 610041) 
Baoying Xia, Xiaofei Wang, Yu Ma, Feng Liu, Yanping Gong, Xiuhe Zou, Jianyong Lei, Anping Su, Tao Wei, Jingqiang Zhu, Zhihui Li

2. Department of Ultrasound, West China Hospital, Sichuan University(Chengdu 610041)

Boyang Yu, Qiang Lu

Corresponding authors

Correspondence to Zhihui Li or Qiang Lu.

Contributions:

Baoying Xia and Boyang Yu wrote the main manuscript text. Xiaofei Wang, Yu Ma, Feng Liu, Yanping Gong, Xiuhe Zou, Jianyong Lei and Anping Su prepared tables 1-5. Tao Wei, Jingqiang Zhu, Qiang Lu and Zhihui Li prepared figures 1-3. All authors reviewed the manuscript.

\section{References}

1. Cheng, Z. and P. Liang, Advances in ultrasound-guided thermal ablation for symptomatic benign thyroid nodules. Advances in clinical and experimental medicine : official organ Wroclaw Medical University, 2020.

2. Durante, C., et al., The Diagnosis and Management of Thyroid Nodules: A Review. JAMA, 2018. 319(9): p. 914-924.

3. Gharib, H., et al., American Association of Clinical Endocrinologists, Associazione Medici Endocrinologi, and European Thyroid Association medical guidelines for clinical practice for the diagnosis and management of thyroid nodules: Executive Summary of recommendations. Journal of endocrinological investigation, 2010. 33(5): p. 287-91.

4. Jiang, L., et al., Variation in the Quality of Thyroid Nodule Evaluations Before Surgical Referral. The Journal of surgical research, 2019. 244: p. 9-14.

5. Khanh, H., et al., Efficacy of Microwave Ablation in the Treatment of Large ( $\geq 3 \mathrm{~cm}$ ) Benign Thyroid Nodules. World journal of surgery, 2020. 44(7): p. 2272-2279.

6. Nixon, I., et al., Image-guided chemical and thermal ablations for thyroid disease: Review of efficacy and complications. Head \& neck, 2018. 40(9): p. 2103-2115.

7. Long, Y., et al., Radiofrequency ablation of liver cancers adjacent to the gallbladder without gallbladder isolation under contrast-enhanced ultrasound monitoring: a preliminary study. International journal of hyperthermia : the official journal of European Society for Hyperthermic Oncology, North American Hyperthermia Group, 2019. 36(1): p. 139-145.

8. YJ, L., et al., Ultrasound-guided microwave ablation in the treatment of benign thyroid nodules in 435 patients. Experimental biology and medicine (Maywood, N.J.), 2017. 242(15): p. 1515-1523. 
9. Papini, E., et al., 2020 European Thyroid Association Clinical Practice Guideline for the Use of ImageGuided Ablation in Benign Thyroid Nodules. European thyroid journal, 2020. 9(4): p. 172-185.

10. Lee, G., et al., Successful radiofrequency ablation strategies for benign thyroid nodules. Endocrine, 2019. 64(2): p. 316-321.

11. Dong, P., et al., The efficacy and safety of microwave ablation versus lobectomy for the treatment of benign thyroid nodules greater than $4 \mathrm{~cm}$. Endocrine, 2020.

12. $\mathrm{Hu}, \mathrm{K}$. , et al., Comparison between ultrasound-guided percutaneous radiofrequency and microwave ablation in benign thyroid nodules. Journal of cancer research and therapeutics, 2019. 15(7): $p$. 1535-1540.

13. Wu, W., et al., US-guided percutaneous microwave ablation for the treatment of benign thyroid nodules. Endocrine journal, 2017. 64(11): p. 1079-1085.

14. Jin, $\mathrm{H}$. , et al., A propensity score matching study between ultrasound-guided percutaneous microwave ablation and conventional thyroidectomy for benign thyroid nodules treatment. International journal of hyperthermia : the official journal of European Society for Hyperthermic Oncology, North American Hyperthermia Group, 2018. 35(1): p. 232-238.

15. Liu, Y., et al., Ultrasound-guided microwave ablation in the treatment of benign thyroid nodules in 435 patients. Experimental biology and medicine (Maywood, N.J.), 2017. 242(15): p. 1515-1523.

16. Yue, W., et al., Ultrasound guided percutaneous microwave ablation of benign thyroid nodules: safety and imaging follow-up in 222 patients. European journal of radiology, 2013. 82(1): p. e11-6.

17. Cesareo, R., et al., Efficacy and safety of a single radiofrequency ablation of solid benign nonfunctioning thyroid nodules. Archives of endocrinology and metabolism, 2017. 61(2): p. 173-179.

18. Heck, K., et al., Percutaneous microwave ablation of thyroid nodules: effects on thyroid function and antibodies. International journal of hyperthermia : the official journal of European Society for Hyperthermic Oncology, North American Hyperthermia Group, 2015. 31(5): p. 560-7.

19. Erturk, M., et al., Microwave ablation of symptomatic benign thyroid nodules: Short-and long-term effects on thyroid function tests, thyroglobulin and thyroid autoantibodies. Clinical endocrinology, 2020.

\section{Figures}




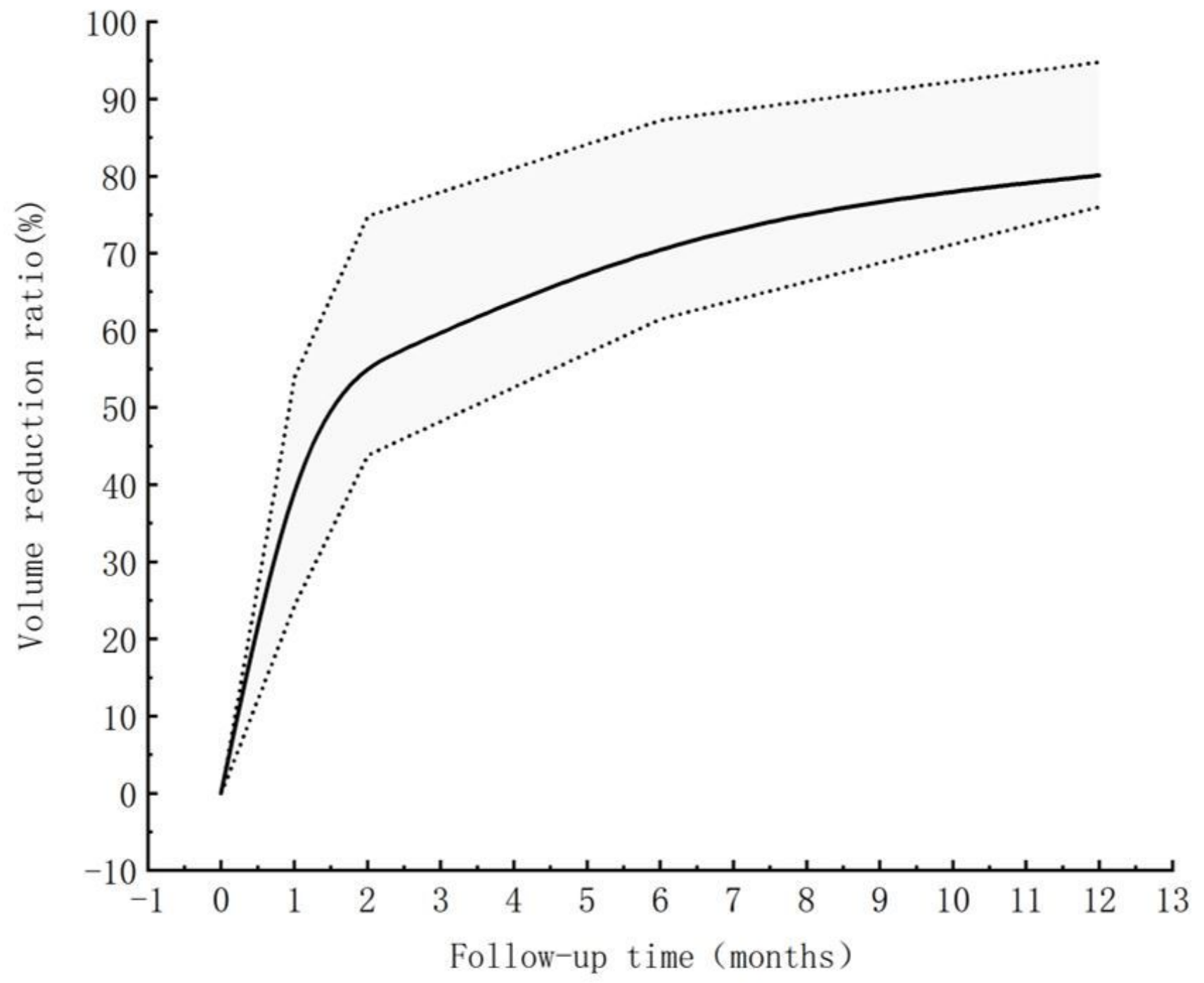

Figure 1

Volume reduction ratio of benign thyroid nodules during the follow-up time. The black line represents the median, and the shaded area represents the discrete variable trend of the volume reduction ratio during follow-up. 


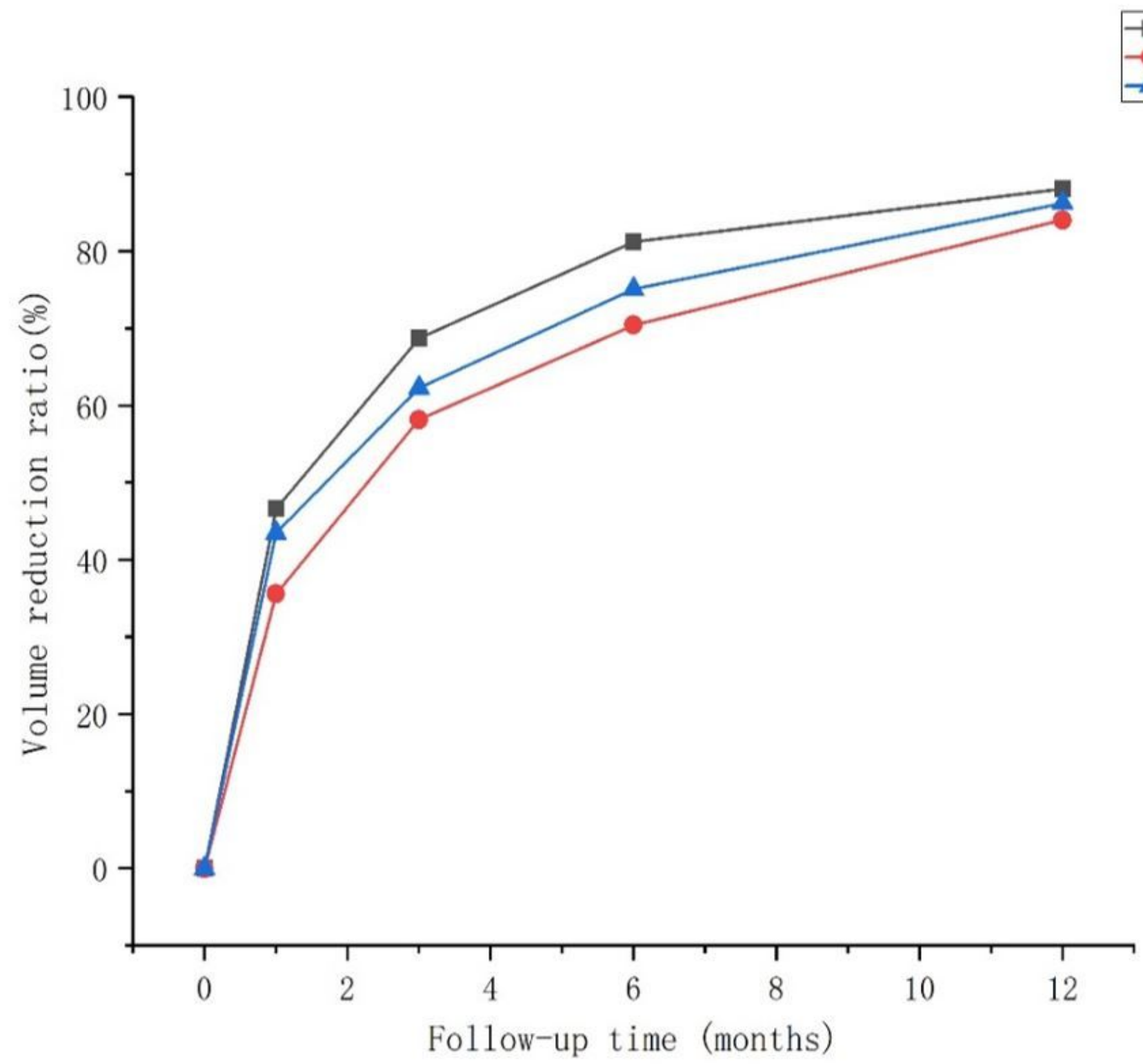

Figure 2

Cystic nodule, solid nodule, and mixed nodule changed in volume reduction ratio $₫ \mathrm{VRR} \otimes$ with the follow-up time. 


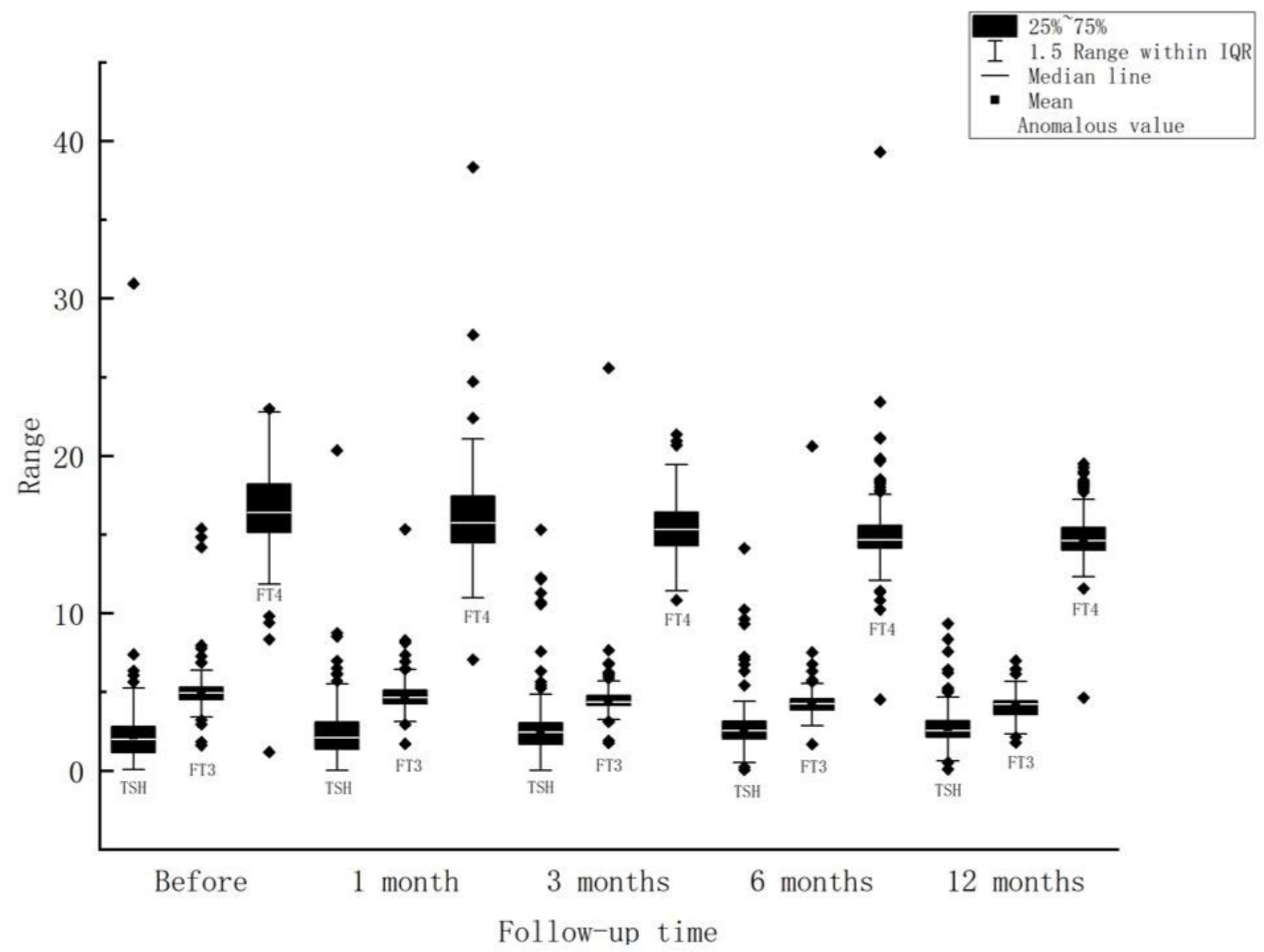

Figure 3

Plasma levels of TSH, FT3 and FT4 in patients with benign thyroid nodules during follow-up at baseline $, 1,3,6$, and 12 months. 